\title{
Soft Palate Benign Granular Cell Tumor
}

National Cancer Institute

\section{Source}

National Cancer Institute. Soft Palate Benign Granular Cell Tumor. NCI Thesaurus. Code C6210.

A rare benign neoplasm that arises from the soft palate. It is characterized by the presence of plump eosinophilic cells with abundant granular cytoplasm. The neoplastic cells extend into the surrounding tissues. Recurrences are rare after removal of the tumor. 